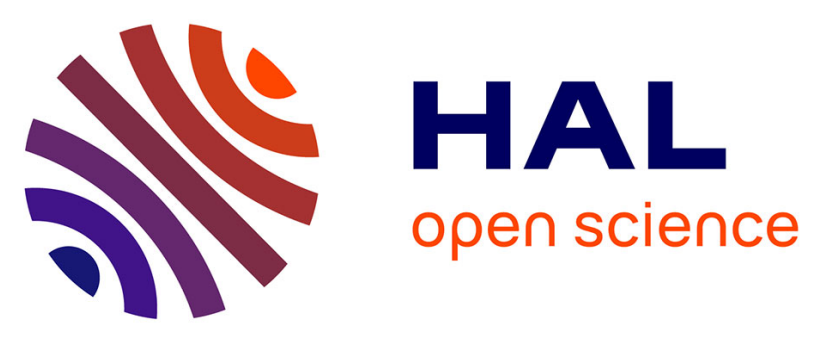

\title{
L'usage de la terre crue dans le mégalithisme des Pyrénées nord-occidentales : nouvelles données
}

Pablo Marticorena, Marylise Onfray, Vincent Ard, Emmanuel Mens, Francis Bichot

\section{- To cite this version:}

Pablo Marticorena, Marylise Onfray, Vincent Ard, Emmanuel Mens, Francis Bichot. L'usage de la terre crue dans le mégalithisme des Pyrénées nord-occidentales: nouvelles données. Bulletin de la Société préhistorique française, 2018, 115 (2), pp.394-397. 10.3406/bspf.2018.14899 . hal-01899820

\section{HAL Id: hal-01899820 \\ https://hal.science/hal-01899820}

Submitted on 19 Oct 2018

HAL is a multi-disciplinary open access archive for the deposit and dissemination of scientific research documents, whether they are published or not. The documents may come from teaching and research institutions in France or abroad, or from public or private research centers.
L'archive ouverte pluridisciplinaire HAL, est destinée au dépôt et à la diffusion de documents scientifiques de niveau recherche, publiés ou non, émanant des établissements d'enseignement et de recherche français ou étrangers, des laboratoires publics ou privés. 


\title{
L'usage de la terre crue dans le mégalithisme des Pyrénées nord-occidentales : nouvelles données
}

\author{
Pablo Marticorena, Marylise Onfray, Vincent Ard, Emmanuel Mens et Francis Bichot
}

\section{Le mégalithisme des Pyrénées nord-occidentales}

Malgré une reconnaissance remontant à la deuxième moitié du XIX ${ }^{\mathrm{e}}$ siècle (Raymond, 1866 par exemple) et un intérêt régulier (Barandiaran, 1953 ; Blot, 1993 ; colloque de la Société d'anthropologie du Sud-Ouest en 1992...), il faut bien reconnaître qu'il est actuellement bien difficile de dresser un portrait précis du phénomène mégalithique des Pyrénées nord-occidentales.

Le regain d'intérêt et surtout les pistes ouvertes par les études actuelles, prônant entre autres des approches prenant en compte les architectures dans leur intégralité ainsi que leur implantation dans le territoire, ouvrent de nouvelles perspectives de recherche.

\section{Nouvelles approches autour de deux fenêtres géographiques}

C'est dans ce contexte que de nouvelles recherches sur le mégalithisme des Pyrénées occidentales ont été engagées en 2015 (Mens et al., à paraître). Ces travaux se sont structurés depuis 2017 sous la forme d'un Projet collectif de recherche (PCR) intitulé « Structures dolméniques et territoires dans les Pyrénées nord-occidentales » (coordination P. Marticorena).

Deux micro-régions ont été sélectionnées comme fenêtres d'étude privilégiées - la vallée d'Hergaray au Pays basque et le plateau de Ger au nord de Lourdes pour questionner l'organisation spatiale et les modes d'occupations des territoires des communautés néolithiques. Ces résultats seront ensuite confrontés aux données recueillies à l'échelle de la région.

Dans ces deux aires d'étude, une première série d'études a été engagée : approche architecturale et technologique des mégalithes, prospections pédestres et géophysiques, reprises de collections anciennes, ou bien encore analyses SIG par exemple. En outre, une série de sondages a pu être réalisée en 2017 sur deux monuments, le dolmen de Gasteenia, sur la commune de Mendive, dans la vallée d'Hergaray, et le tumulus S, commune d'Azereix, sur le plateau de Ger. Les études géoarchéologiques préliminaires permettent la reconnaissance de l'usage de la terre crue pour l'édification des tertres funéraires.

\section{Gasteenia, une construction unique}

Gasteenia est sans doute l'un des sites mégalithiques les plus emblématiques du Pays Basque. Il est mentionné pour la première fois en 1945 par J.M. Barandiaran et classé au titre des Monuments Historiques en 1952. Par la suite, il sera mis en avant dans de nombreux ouvrages grand public, sans aucune mention d'intervention archéologique, et le seul plan disponible est celui publié par Y. Chevallier en 1987. L'étude préliminaire, en 2016, était donc destinée à mener une première analyse architecturale et pétrographique et à dresser un nouveau relevé du monument.

On constate l'investissement porté sur la chambre mégalithique, par l'emploi de roches soigneusement sélectionnées, mettant en scène une dalle de chevet anthropomorphe (Mens et al., à paraître). Lors de cette étude, nous avions aussi été particulièrement interpellés par la position de ce dolmen au sommet d'une butte qui semblait peu compatible avec une formation naturelle. La vérification de cette hypothèse constituait alors l'objectif principal des sondages menés en 2017.

Parmi les quatre sondages réalisés, les sondages 2 et 4 ont permis de disposer d'une coupe complète de cette butte supportant la chambre mégalithique. Elle présente une succession d'unités stratigraphiques dont la principale correspond à une accumulation d'éléments subarrondis ou subanguleux argilo-sableux de nature hétérogène (variabilité dans la nature et la proportion des constituants ainsi que la couleur) ce qui permet de confirmer le caractère anthropique de ce relief (fig 1). L'observation fine de la stratigraphie archéologique permet d'interpréter ces éléments comme des mottes de terre crue. L'hypothèse retenue actuellement est la présence d'un tertre de terre façonné en bauge (bauge en motte et bauge litée, voir Onfray, 2017), associant parfois des niveaux de pierres. Cette hypothèse doit être confirmée par une analyse microstratigraphique à partir de l'analyse micromorphologique des sols. De même, cette hypothèse ouvre de nombreuses questions concernant les techniques architecturales engagées.

La question des techniques constructives pour l'aménagement des tertres funéraires a été abordée pour d'autres sites. La technique en motte de gazon a été reconnue sur plusieurs tumulus néolithiques : " la Butte du Houx » à Sarceaux (Chancerel et Desloges, 1998), « le Cruchaud» à Saint-Heurine (Berthet et Ferrier, 2003) et plus récemment « les Hauts de l'Orne » à Fleury-sur-Orne (Ghesquière et al., 2015). Dans des contextes pédologiques où ce type de ressource n'est pas présent, les techniques constructives employées sont autres et relèvent plutôt de la bauge. Cette technique est «plus élaborée » dans le sens où elle requiert une préparation des matériaux (mélange, adjonction d'eau), voire des ajouts. Cependant, nos connaissances sont pour l'heure limitées à l'emploi de la bauge pour la construction des tertres funéraires, car 

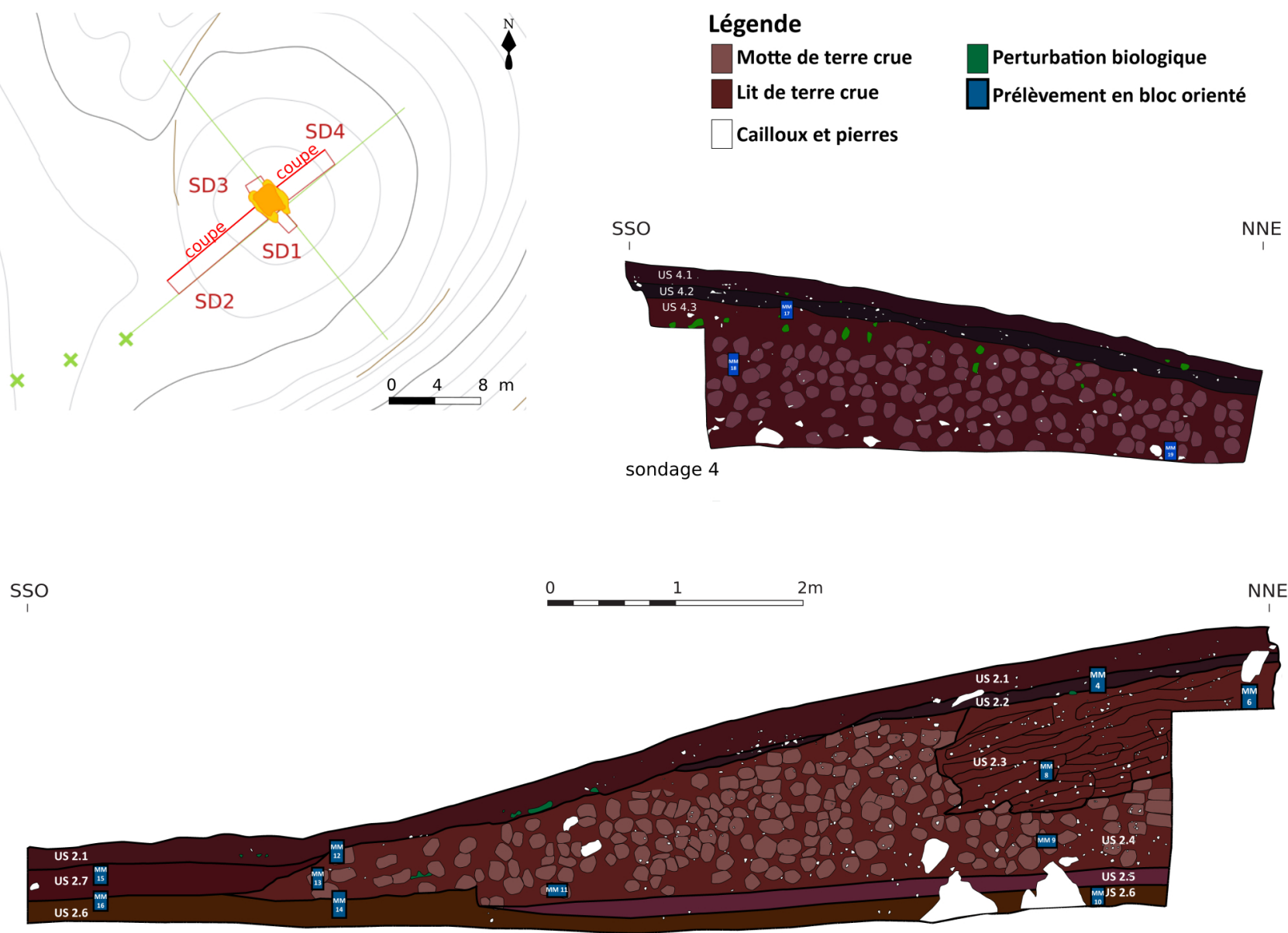

sondage 2

Fig. 1 - Plan et coupe du site de Gasteenia (relevé et DAO : V.-E. Leroux, A. Laurent, M. Onfray, P. Marticorena).

nous ne disposons que de deux exemples. Il s'agit, dans un cas, du site néolithique de Chuso Nova I en Galice où le tertre, conservé sur près d'un mètre de hauteur, est constitué de «boules de terre » de couleurs différentes (Bóveda Fernandez et Vilaseco Vásquez, 2015). Dans l'autre cas, il s'agit du tumulus du Chemin de la Lande à Claracq (Pyrénées-Atlantiques), conservé sur $0,50 \mathrm{~m}$ de hauteur atteste de la présence de terre crue (Wattez, in Chopin et Allenet de Ribemont, 2013). Cependant, ces deux sites ne disposent pas d'un développement de la stratigraphie aussi important que le site de Gasteenia puisque celle-ci est d'une puissance de près de $2 \mathrm{~m}$, et confère alors au site un caractère singulier.

En définitive l'appellation « dolmen simple pyrénéen » couramment admise pour les sites de notre région, masque des architectures de formes diverses, avec parfois une mise en scène jouant sur les volumes, les pentes et les couleurs des monolithes. Elle est particulièrement exacerbée dans le cas de Gasteenia par l'érection d'une chambre mégalithique au sommet d'une butte artificielle.

\section{Le tumulus $S$, un monument complexe}

Le tumulus S, localisé sur le plateau de Ger au sein d'une grande nécropole tumulaire de plus de 200 monuments a été fouillé par E. Pothier en 1884 (Pothier, 1892). Aucune autre opération n'est connue par la suite. Les premiers relevés laissaient déjà entrevoir un monument imposant possédant un tertre à l'architecture complexe.

$\mathrm{Au}$ cours de notre étude, treize monolithes ont été documentés. La variété géologique est remarquable (différentes variétés de grès, granite, calcaire et quartzite) et implique des transports compris entre 3 et $10 \mathrm{~km}$ et donc un investissement en énergie considérable pour l'acquisition de certains de ces blocs.

Un seul sondage de faible superficie $\left(1,9 \mathrm{~m}^{2}\right)$ a été pratiqué, installé sur la paroi nord-ouest du cône de fouille du $\mathrm{XIX}^{\mathrm{e}}$ siècle. La stratigraphie relevée a également permis d'attester l'utilisation de la terre crue. Audessus d'une US identifiée à la base du sondage (US4), interprétée comme un apport volontaire de pierres et de terre dont la nature reste à préciser, un niveau de terre crue (US3) a été identifié (fig 2). Cette couche présente en effet plusieurs critères d'identification de la terre crue reconnue dans différents contextes (Wattez, 2009 ; Wattez et Onfray, 2014 ; Onfray, 2017), à savoir un mélange de matériaux de nature différente, une forte compaction et une structure complexe. Par ailleurs, le détachement des agrégats sous la forme de pans souligne l'idée d'apports de terre successifs.

Ce monument présente donc une complexité architecturale et un investissement technique tout à fait remarquables aussi bien pour la chambre mégalithique que pour l'édification du tumulus comprenant un probable 

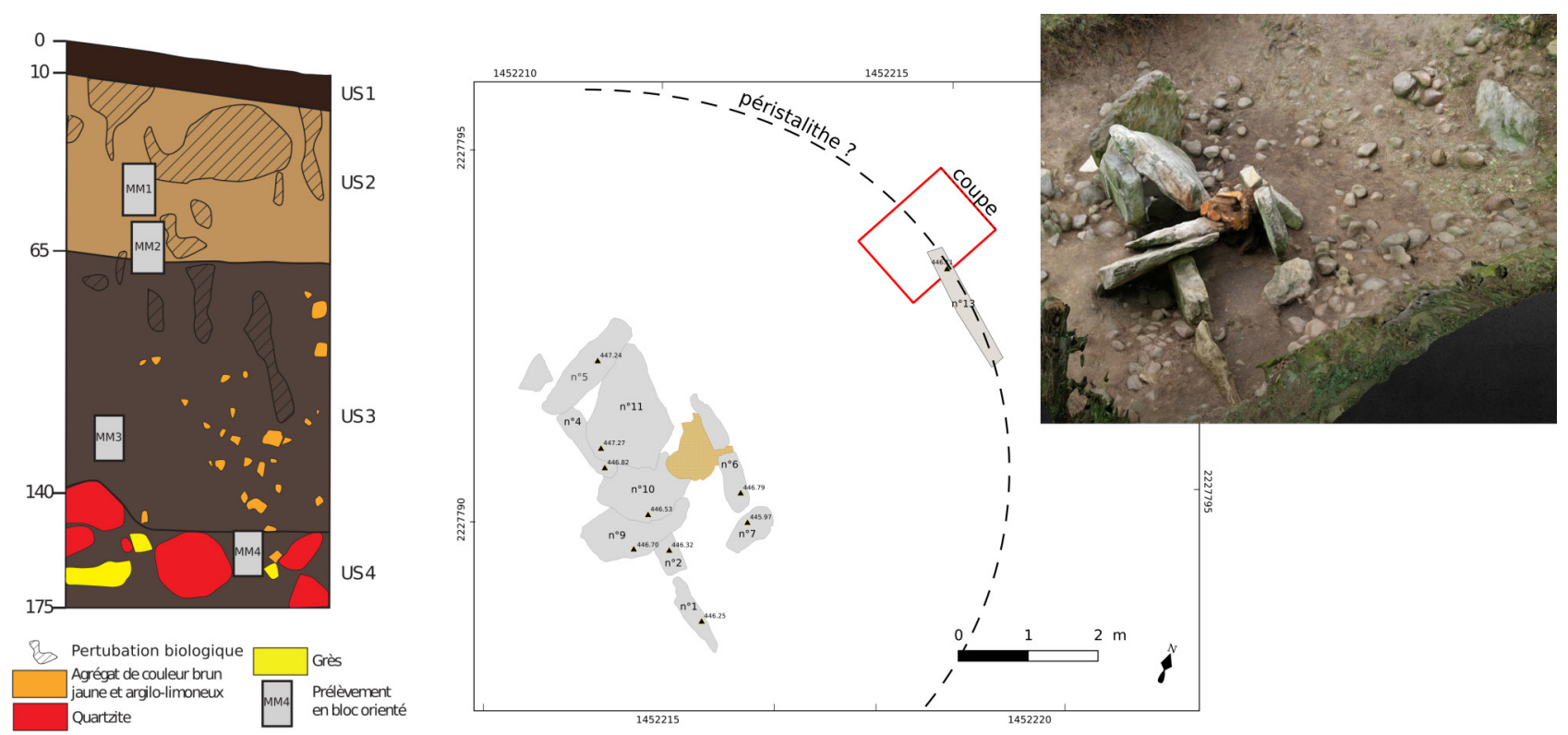

Fig. 2 - Plan, coupe et photogrammétrie du tumulus S (relevé et DAO : V.-E. Leroux, A. Laurent, M. Onfray, P. Marticorena).

péristalithe interne retenant des galets et une structure en terre crue sur la partie extérieure de ce péristalithe. Cette architecture pose également la question d'un phasage de construction qui reste à déterminer.

\section{Une reconnaissance marquante pour les études mégalithiques}

Ces premières études montrent une utilisation de la terre crue dans les structures funéraires mégalithiques des Pyrénées occidentales aussi importante qu'inattendue pour un matériau jusqu'alors surtout réservé à la sphère domestique (Sénépart et al., 2015) jusque dans des régions septentrionales comme la Bretagne avec le site d'habitat de Lillemer en Ille-et-Vilaine (Laporte et al. 2015). L'enjeu dorénavant est de mieux appréhender comment l'architecture en terre participe à l'émergence du monumentalisme dans les Pyrénées nord-occidentales. Le site de Gasteenia apparaît comme un site particulièrement bien conservé qui pourrait devenir une référence pour cette question, d'autant plus qu'il est actuellement impossible de déterminer si la butte de terre sur laquelle repose le dolmen était funéraire ou non. La suite de nos travaux cherchera donc à préciser ces questions de phasage de construction, de modalités d'installation (terrassements, nivellements ?) et de chaîne opératoire de mise en œuvre de la terre crue (origine des matériaux, modes de préparation, modes de mises en œuvre). Enfin, il faudra s'interroger sur l'évolution de ces tertres au cours du temps et sur les processus post-dépositionnels mis en jeu permettant ou non leur conservation.

\section{RÉFÉRENCES BIBLIOGRAPHIQUES}

Barandiaran J.M. DE (1953) - El Hombre Prehistorico en el Pais Vasco, Buenos Aires, Editorial Vasca Ekin, 267 p.
Berthet A.-L., Ferrier C. (2003) - Le tumulus du Cruchaud à Sainte-l'Heurine, Charente-Maritime -II- Apports de l'étude micromorphologique, Bulletin de la Société préhistorique française, 100, 2, p. 293-322.

Bóveda Fernandez J., Vilaseco Vásquez X. I. (2015) - La cámara megalítica de Chousa Nova 1 (Silleda, Pontevedra): ¿Rotura intencional o colapso?, in V.S. Gonçalves, M. Diniz, A.C. Sousa dir., $5^{\circ}$ Congresso do Neolitico Peninsular, Lisboa, Centro de Arqueologia da Universidade de Lisboa (coll. Estudo \& Memórias, 8), p. 564-570

Blot J. (1993) - Archéologie et montagne basque, San Sebastian, Elkar, $240 \mathrm{p}$.

Chancerel A., Desloges J. (1998) - Les sépultures prémégalithiques de Basse-Normandie, in J. Guilaine dir., Sépultures d'Occident et genèse des mégalithismes (9000-3500 av. n.è.) : séminaire du Collège de France, Paris, Errance, p. $96-106$.

Chevallier Y.(1987) - À propos des monuments dolméniques du Pays Basque, in Les hommes et leurs sépultures dans les Pyrénées occidentales, depuis la préhistoire, Catalogue de l'exposition Archéologie des Pyrénées Occidentales et des Landes, s.1., s.n., tome 7, p.142-148.

Chopin J.-F., Allenet De Ribemont G. (2013) - A65 - Claracq (64), Chemin de la Lande : rapport de fouilles, Rapport final d'opération de fouille préventive, Bordeaux, Inrap Grand Sud-Ouest/Service régional de l'archéologie, 133 p.

Ghesquiere E., Giazzon D., Wattez J. (2015) - Construction en terre crue au Néolithique moyen I, le tertre du monument 29 de Fleury-sur-Orne, Archéopages, 42, p. 28-35.

Laporte L., Bizien-Jaglin C., Wattez J., Barreau J.b., Bernard Y., Aoustin D., GuitTo V., Hamon G., JaLlot L., Lucquin A., March R., Marcoux N., Mens E., Soler L., Werthe E. (2015) - Another brick in the wall : fifth millenium $\mathrm{BC}$ earthen-walled architecture on the Channel shores, Antiquity, vol. 89, issue 346, p. 800-817. 
Mens E., Marticorena P., Ard V., Boscus S., Caule B., LiNARD D., BichOT F. (à paraître) - Regards croisées sur le mégalithisme de la Méditerranée à l'Atlantique : le Pays Basque et ses marges, in Marticorena et al. (dir.), Entre deux mers, actualité de la recherche, Actes des $12^{e}$ rencontres méridionales de Préhistoire récente, Bayonne, 27 septembre-1 ${ }^{\text {er }}$ octobre 2016, Toulouse, AEP.

Onfray M. (2017) - Du sol à la reconstitution de l'espace habité : géoarchéologie des modes d'occupation de la fin du Néolithique (3600-2250 av. n.-è.) dans le Sud-Ouest du Bassin parisien, Thèse de doctorant, université Paris 1 Panthéon-Sorbonne, Paris, 673 p.

Pothier E. (1892) - Tumulus-dolmen de Marque-Dessus (commune d'Azereix, Hautes-Pyrénées), Anthropologie, p. $37-42$.

Raymond P. (1866) - Notice sur le dolmen d'Escout (BassePyrénées), Communication de M. P. Raymond, correspondant, Revue des sociétés savantes, $4^{\mathrm{e}}$ série, t. IV, p. 500-501.

Senepart I., Wattez J., Jallot L., Hamon T., Onfray M. (2015) - La construction en terre crue au Néolithique : un état de la question en France, Archéopages, 42, p. 7-19.

WATteZ J. (2003) - Caractérisation micromorphologique des matériaux façonnés en terre crue dans les habitats néolithiques du Sud de la France : exemple des sites de Jacques Coeur (Montpellier, Hérault), du Jas del Biau (Miliau, Aveyron), la Capoulière (Mauguoi, Hérault), in C.-A. Chazelles et A. Klein (dir.), Échanges transdisciplinaires sur les constructions en terre crue-1- Terre modelée, découpée ou coffrée : matériaux et modes de mise en oeuvre, Actes de la table ronde de Montpellier, 17-18 novembre 2001, Montpellier, Espérou, p. 21-31.

WATtez J. (2009) - Enregistrement sédimentaire de l'usage de la terre crue dans les établissements néolithiques du Sud de la France : le cas des sites du Néolithique final de La Capoulière 2 et du Mas de Vignoles IV, in A. Beeching et I. Sénépart (dir.), De la maison au village : l'habitat néolithique dans le Sud de la France et le Nord-Ouest méditerranéen, Actes de la table ronde des 23 et 24 mai 2003, Marseille/ Musée d'Histoire de la Ville de Marseille, Paris, Société préhistorique française (coll. Mémoires, 48), p. 199-218.

Wattez J., Onfray M. (2014) - La question des sols d'occupation néolithiques : apports de la géoarchéologie à leur identification et à leur interprétation, in I. Sénépart C. Billard, F. Bostyn, I. Praud, E. Thirault (dir.), Méthodologie de terrain sur la Préhistoire récente en France : nouveaux acquis, nouveaux outils, 1987-2012, Actes des premières rencontres de Préhistoire récente Nord/Sud, Marseille, 23-25 mai 2012, Toulouse, AEP, p. 317-331.

Pablo Marticorena

Docteur en archéologie

UPPB-EHUH

UMR 5608 TRACES

Université de Toulouse Jean-Jaurès

Maison de la Recherche

5, allées Antonio-Machado

31058 Toulouse Cedex 9

harriak@hotmail.fr

Marylise OnfraY

Post-doctorante UMR 8215 Trajectoires

UFR DMOS-Agroparistech

Maison Archéologie Ethnologie René-Ginouvès 21, allée de l'Université

92023 Nanterre Cedex

Vincent ARD

Chargé de recherche CNRS UMR 5608 TRACES

Emmanuel Mens

Docteur en archéologie UMR 5608 TRACES

Francis BICHOT

Docteur en géologie 